\title{
Genetic Deletion of Galectin-3 Alters the Temporal Evolution of Macrophage Infiltration and Healing Affecting the Cardiac Remodeling and Function after Myocardial Infarction in Mice
} Check for updates

\author{
Pablo Cassaglia, ${ }^{\star}$ Federico Penas, ${ }^{\dagger}$ Celeste Betazza, ${ }^{*}$ Florencia Fontana Estevez, ${ }^{\ddagger}$ Verónica Miksztowicz, \\ Nadia Martínez Naya, ${ }^{*}$ María Clara Llamosas, ${ }^{*}$ Sofía Noli Truant, ${ }^{\top}$ Luciana Wilensky, ${ }^{*}$ Verónica Volberg, ${ }^{*}$ Ágata C. Cevey,

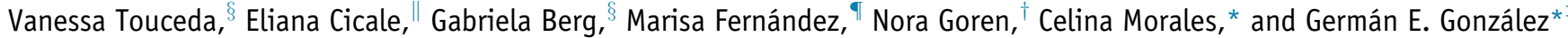

\begin{abstract}
From the Facultad de Medicina-CONICET, * Departamento de Patología, Instituto de Fisiopatología Cardiovascular, the Facultad de Medicina, ${ }^{\dagger}$ Instituto de Investigaciones Biomédicas en Retrovirus y SIDA (INBIRS), the Facultad de Farmacia y Bioquímica, ${ }^{\S}$ Departamento de Bioquímica Clínica-INFIBIOC, Laboratorio de Lípidos y Aterosclerosis, the Facultad de Farmacia y Bioquímica-CONICET, Instituto de Estudios de la Inmunidad Humoral (IDEHU), and the Facultad de Ciencias Veterinarias, "Universidad de Buenos Aires, Buenos Aires; and the Facultad de Medicina, ${ }^{\ddagger}$ Pontificia Universidad Católica Argentina (UCA), Instituto de Investigaciones Biomédicas (UCA-CONICET), Laboratorio de Patología Cardiovascular Experimental e Hipertensi Arterial, Buenos Aires, Argentina
\end{abstract}

Accepted for publication

May 5, 2020.

Address correspondence to Germán E. González, M.D. Ph.D., J. E. Uriburu 950, Piso 2, C1114AAD Buenos Aires, Argentina. E-mail: germangonzalez@uca.edu.ar.

\begin{abstract}
We studied the role of galectin-3 (Gal-3) in the expression of alternative activation markers (M2) on macrophage, cytokines, and fibrosis through the temporal evolution of healing, ventricular remodeling, and function after myocardial infarction (MI). C57BL/6J and Gal-3 knockout mice (Lgals3 ${ }^{-1}$ ) were subjected to permanent coronary ligation or sham. We studied i) mortality, ii) macrophage infiltration and expression of markers of alternative activation, iii) cytokine, iv) matrix metalloproteinase-2 activity, v) fibrosis, and vi) cardiac function and remodeling. At 1 week post-MI, lack of Gal-3 markedly attenuated $\mathrm{F} 4 / 80+$ macrophage infiltration and significantly increased the expression of Mrc1 and Chil1, markers of M2 macrophages at the MI zone. Levels of IL-10, IL-6, and matrix metalloproteinase-2 were significantly increased, whereas tumor necrosis factor- $\alpha$, transforming growth factor- $\beta$, and fibrosis were remarkably attenuated at the infarct zone. In Gal-3 knockout mice, scar thinning ratio, expansion, and cardiac remodeling and function were severely affected from the onset of MI. At 4 weeks post-MI, the natural evolution of fibrosis in Gal-3 knockout mice was also affected. Our results suggest that Gal-3 is essential for wound healing because it regulates the dynamics of macrophage infiltration, proinflammatory and anti-inflammatory cytokine expression, and fibrosis along the temporal evolution of MI in mice. The deficit of Gal-3 affected the dynamics of wound healing, thus aggravating the evolution of remodeling and function. (Am J Pathol 2020, 190: 1789-1800; https://doi.org/10.1016/ j.ajpath.2020.05.010)
\end{abstract}

Myocardial infarction (MI) leads to a sequence of structural and functional changes that promote the healing of the infarct zone, ventricular remodeling (VR), and myocardial decompensation. ${ }^{1}$ The temporal evolution of wound healing is associated with the activation of a well-orchestrated and balanced inflammatory response that leads to the formation of a fibrous scar, ${ }^{2}$ which should provide a satisfactory tension force to prevent infarct expansion ${ }^{3}$ and adverse remodeling.

\footnotetext{
Supported by National Agency for Promotion of Science and Technology grants PICT 2014-2320 (G.E.G.) and PICTO 2017-055 (G.E.G.) and University of Buenos Aires, Argentina; grant UBACyT 20020170100619BA (G.E.G.).

F.P., V.M., Á.C.C., G.B., M.F., N.G., and G.E.G. are members of the Investigator Career from the National Council of Scientific and Technical Research (CONICET) of Argentina; F.F.E. and L.W. are fellows from the CONICET of Argentina.

Disclosures: None declared.
} 
Nevertheless, throughout the temporal evolution of wound healing, the progression of MI to heart failure is also linked to a multiplicity of interrelated factors associated with adverse remodeling. ${ }^{4,5}$ Although the mechanisms responsible for the evolution of post-MI VR are multiple, diverse, and complex, the persistent increased activation of the inflammatory response triggered during the repair of the infarct zone notably contributes to the adverse evolution of left ventricular (LV) structural changes and failure. ${ }^{6}$ Recent studies have found that monocyte and macrophage subpopulations recruited at the infarct zone exhibit a biphasic functional heterogeneity, mainly orchestrated by the infiltration of M1 and M2 macrophages. ${ }^{7-9}$ Thus, monocytes and macrophages promote a well-organized clearance of necrotic cells and facilitate the repairing process by collagen synthesis. ${ }^{10-13}$ Largely, macrophages may also determine the evolution of cardiac remodeling because exaggerated inflammation may also affect the healing as seen by the increase in mortality of hospitalized patients with MI. ${ }^{4,14}$ Therefore, targeting the healing to improve MI consequences has proven to be ambiguous $^{15}$ because insufficient or disproportionate inflammation at early stages of MI or limited or exaggerated fibrosis at later stages will depend on temporal and spatial factors.

Macrophages and fibroblasts that infiltrate the infarct zone are the major sources of galectin-3 (Gal-3). Gal-3 is a $\beta$-galactosidase-binding lectin widely expressed in the immune system and markedly involved in cardiovascular pathophysiology as a proinflammatory and profibrotic molecule. ${ }^{16,17}$ During the past few years, this lectin merged as a strong prognostic factor in patients with coronary artery disease and heart failure. ${ }^{18}$ In rodents, the synthesis of Gal-3 highly increased during the first 2 weeks after MI, suggesting it is a regulatory factor in the regulation of wound healing. ${ }^{17,19}$ Despite some essential cellular and molecular factors along the temporal evolution of wound healing and their pathophysiologic significance on cardiac remodeling, the role of Gal-3 as a key factor in the temporal evolution of macrophage infiltration, tissue repair, remodeling, and function is still unknown. We hypothesized that Gal-3 regulates macrophage infiltration, the expression of markers of its alternative activation, cytokine expression, and fibrosis along the temporal evolution of wound healing and thus contributes to the evolution of VR and cardiac dysfunction in mice.

\section{Materials and Methods}

\section{Mice and Experimental Models of MI}

Male C57BL/6J and Gal-3 knockout (KO) $\left(\right.$ Lgals $\left.^{-/-}\right)$mice (8 to 10 weeks old) were used. C57BL/6J mice were purchased from the Department of Bioresources of the Faculty of Veterinary Sciences of the University of Buenos Aires, Buenos Aires, Argentina, and used as controls. Gal-3 KO mice were originally donated by Dr. Gabriel A. Rabinovich
[Laboratorio de Inmunopatología, Instituto de Biología y Medicina Experimental (IBYME-CONICET), Buenos Aires, Argentina] and were bred on a C57BL/6J background at the same bioresources facilities stated above. Mice were anesthetized with intramuscular administration of ketamine $(65 \mathrm{mg} / \mathrm{kg})$, xylazine $(13 \mathrm{mg} / \mathrm{kg})$, and acepromazine $(1.5$ $\mathrm{mg} / \mathrm{kg}$ ) and then intubated and given mechanical ventilatory support using a Harvard ventilator (tidal volume, $250 \mu \mathrm{L}$ per stroke) at a respiratory frequency of 34 to 38 cycles per minute, as described previously. ${ }^{20}$ Subsequently, lateral left thoracotomy was performed followed by pericardiectomy and ligature of a lateral branch of the left descending coronary artery using an 8.0 silk thread. Finally, the chest was closed by layers, and the animals were allowed to recover from the anesthesia in a quiet environment. Animals in the sham operation group underwent the same procedure without ligature of the coronary artery. Finally, when animals recovered from anesthesia, they were housed in individual cages until the end of the protocol. All the experiments were approved by the Animal Care and Research Committee of the University of Buenos Aires in line with the NIH's Guide for the Care and Use of Laboratory Animals. ${ }^{21}$

Mice were randomized according to the evolution time into the following groups: i) C57 sham, ii) Gal-3 KO sham, iii) C57 MI, iv) Gal-3 KO MI for 1 week v) C57 sham, vi) Gal-3 KO sham, vii) C57 MI, and viii) Gal-3 KO MI for 4 weeks.

\section{Echocardiography}

Echocardiography was performed at 1 and 4 weeks after surgery in anesthetized animals (tribromoethanol; 1.15 $\mathrm{mL} / \mathrm{kg}$ ). LV dimensions (wall thickness, cavity size, and areas in systole and diastole) and ventricular function (ejection fraction, shortening fraction, and cardiac output) were evaluated with a Doppler echocardiography system equipped with a $12-\mathrm{MHz}$ linear transducer (Acuson, Mountain View, CA). Cardiac mass was also assessed as previously described. ${ }^{16}$

\section{Histologic Analysis}

Randomized hearts from each group were subjected to histologic examination (4 to 9 hearts per group). Hearts were harvested and immersed in $10 \%$ formaldehyde for 72 hours. Later, they were cut from apex to base and embedded in paraffin; 5-mm serial cuts were made, and sections were stained with hematoxylin and eosin, Masson trichrome, and Picrosirius red. In the sections, also stained with Picrosirius red, interstitial collagen was measured in the septum and scar using the image-analysis software (Image Pro-Plus version 6.0, Media Cybernetics, Silver Spring, MD). The percentage of collagen for each region was calculated by adding the areas corresponding to collagen and dividing by the addition of the areas corresponding to myocyte plus the 
areas of collagen tissue as described elsewhere. ${ }^{16,22}$ Myocyte cross-sectional areas were determined on digitalized images of rhodamine-conjugated lectin-stained sections (WGA RL-1022, Vector Laboratories, Burlingame, CA) of paraffin-embedded samples. These digitalized images were obtained using a fluorescence microscope (Olympus BX61, Olympus, Tokyo, Japan) attached to a digital camera and connected to a computer equipped with Image Pro-Plus version 6.0. Outlines of myocyte were traced, and cell areas were measured with Image Pro-Plus version 6.0.2

\section{Quantitative Determination of Infarct Size and Morphometry}

Myocardial infarct size, wall thickness, and length of endocardial and epicardial circumferences were measured by planimetry from histologic images of the stained sections with Masson trichrome using the Image Pro-Plus version 6.0 software. Infarct size was calculated as the total length of the scar using the mean endocardial and epicardial tracings as a percentage of the total $\mathrm{LV}$ circumference. ${ }^{22}$ The scar thickness (mean of five equidistant measurements) and septum thickness (mean of three equidistant measurements) were measured in a middle section of the heart. The scar thickness ratio was calculated as the association of scar thickness and septum thickness with the expansion index expressed as LV cavity area/total LV area $\times$ septum thickness/scar thickness. ${ }^{23}$ In a pilot experiment, a subset of $\mathrm{C} 57$ and Gal-3 KO mice $(n=5$ per group) underwent permanent coronary artery ligation at 24 hours, followed by immediate euthanasia; hearts were perfused with Evan's blue and then cut from apex to base. The sections were incubated in $1 \%$ of triphenyl tetrazolium chloride isotonic phosphate buffer $(\mathrm{pH}$ 7.4) (Sigma-Aldrich, St. Louis, MO) staining for measurement of infarct size as described previously. ${ }^{24}$ Thus, the infarct size was quantified at 24 hours after permanent coronary artery ligation to ensure that initial injury was comparable between groups.

\section{Flow Cytometry}

Hearts of 1-week infarcted mice (wild type or Gal-3 KO) were processed to obtain single-cell suspensions by flow cytometry. First, MI and LV tissues were harvested by dissection and chopped into small pieces. A mechanical disaggregation method, using pipette and steel mesh, was performed, using phosphate-buffered saline (PBS) as buffer. Cells were then recovered and washed with fresh PBS. After refrigerated centrifugation at $500 \times g$ for 5 minutes, pellets were resuspended in PBS and incubated with red blood cell lysis buffer $(155 \mathrm{mmol} / \mathrm{L}$ ammonium chloride, $12 \mathrm{mmol} / \mathrm{L}$ sodium bicarbonate, $0.1 \mathrm{mmol} / \mathrm{L}$ EDTA, $\mathrm{pH} 7.3$ ). After 20 minutes on ice, the reaction was stopped by the addition of PBS. Cells were centrifuged at $500 \times g$ for 5 minutes and then washed once again in PBS. Thus, 0.3 to $2 \times 10^{6}$ cells were placed in FACS tubes. Then, all samples were blocked with $10 \%$ mouse serum for 30 minutes on ice. This was followed by staining all samples for 30 minutes on ice with anti-mouse F4/80 conjugated with phosphatidylethanolamine (eBioscience, San Diego, CA) at the appropriate dilution (in PBS and 1\% fetal bovine serum) in line with the manufacturer's guidelines. Cells were then washed in $1 \%$ fetal bovine serum and PBS before acquisition and analysis. Data were collected in a PARTEC PAS IIIcytometer (Sysmex Partec GmgH, Görlitz, Germany) and analyzed using Flowing Software (Turku Bioimaging, Turku, Finland) and CellQuest Pro software (BD Biosciences, Franklin Lakes, $\mathrm{NJ}$ ) to determine the percentage of infiltrated macrophages at both MI and remote zone.

\section{Zymography}

Matrix metalloproteinase (MMP)-2 activity from the infarct zone was measured by gelatinolitic zymography. Briefly, cardiac tissue was homogenized in $50 \mathrm{mmol} / \mathrm{L}$ Tris buffer, $\mathrm{pH} 7.4$, containing $5 \mathrm{mmol} / \mathrm{L}$ calcium chloride, $1 \mathrm{mmol} / \mathrm{L}$ zinc chloride, and $1 \%$ Triton X-100. Protein $(120 \mu \mathrm{g})$ was applied to nonreduced SDS-PAGE using 7.5\% gel copolymerized with gelatin $0.1 \%$ (G-8150, Sigma-Aldrich). Gels were run 3 hours in $25 \mathrm{mmol} / \mathrm{L}$ Tris, $192 \mathrm{mmol} / \mathrm{L}$ glycine, $0.1 \%$ SDS at $4^{\circ} \mathrm{C}, \mathrm{pH} 8.3$, in a Mini Protean-3 (BioRad Laboratories, Hercules, CA). After running, gels were rinsed with $2.5 \%$ Triton $\mathrm{X}-100$ for 30 minutes and then incubated for 18 hours in $0.15 \mathrm{~mol} / \mathrm{L}$ sodium chloride, $10 \mathrm{mmol} / \mathrm{L}$ calcium chloride, Tris hydrochloride, $\mathrm{pH} 7.4$, at $37^{\circ} \mathrm{C}$. After staining with Coomassie blue R-250 (B-0149, SigmaAldrich) and destaining with acetic acid, methanol, and water (1:3:6); enzyme activity was detected as colorless bands against the blue-stained background. MMP-2 (67 $\mathrm{kDa}$, active form) and MMP-9 (84 kDa, active form) were identified by molecular weight. Conditioned media from the promyelocyte U-937 cell lines were used as a standard activity. Coefficients of variation were $4.8 \%$ (intra-assay) and $8.6 \%$ (interassay). Band intensities were quantified using ImageJ software (NIH, Bethesda, MD; http://imagej.nih. gov/ij), and relative activity was expressed as a ratio to the internal standard.

\section{Quantitative Real-Time RT-PCR}

Total RNA was extracted from heart tissue homogenates by using a QuickZol reagent (Kalium Technologies, Buenos Aires, Argentina). Total RNA was reverse transcribed using Expand Reverse Transcriptase (Invitrogen Corp., Waltham, MA). Quantitative real-time PCR was performed using a $5 \times$ HOT FIREPol EvaGreen qPCR Mix Plus (ROX) (Solis BioDyne Corp., Tartu, Estonia) in a StepOne System. Primer sequences were as follows: tumor necrosis factor Tnf $(T N F-\alpha)$ forward: 5'-ATGAGCACA GAAAGCATGATC- $3^{\prime}$, reverse: 5'-TACAGGCTTGT CACTCGAATT-3'; IL-6 (also known as Il6) forward: 
5'-TGATGCACTTGCAGAAAACAA-3' ${ }^{\prime}$, reverse: $5^{\prime}$-GGT CTTGGTCCTTAGCCACTC-3'; Ym1 (also known as Chill), forward: 5'-GGATGGCTACACTGGAGAAA-3', reverse: 5'-AGAAGGGTCACTCAGGATAA-3'; mannose receptor (CD206, also known as Mrcl) forward: 5' CAAGGAAGGTTGGCATTTGT- $3^{\prime}$, reverse: $5^{\prime}$-CCTTT CAGTCCTTTGCAAGC-3'; IL-10 (also known as Il10) forward $\quad 5^{\prime}$-CTCCCCTGTGAAAATAAGAGCA-3'; reverse $5^{\prime}$-TCCAGCAGACTCAATACACACT-3'; and transforming growth factor (Tgfb1, also known as $T G F-\beta$ ): forward 5'-CACCGGAGAGCCCTGGATA-3'; reverse 5'TGTACAGCTGCCGCACACA-3'. All samples were analyzed in the same run for $18 \mathrm{~S}$ expression for normalization: forward: $5^{\prime}$ AACACGGGAAACCTCACCC- $3^{\prime}$ and reverse: $5^{\prime}$-CCACCAACTAAGAACGGCCA-3'. PCR parameters were $52^{\circ} \mathrm{C}$ for 2 minutes, $95^{\circ} \mathrm{C}$ for 15 minutes, and 40 cycles of $95^{\circ} \mathrm{C}$ for 30 seconds and $60^{\circ} \mathrm{C}$ (for IL-6, mannose receptor, IL-10, TNF- $\alpha$, and $18 \mathrm{~S}$ ) or $63^{\circ} \mathrm{C}$ (for TGF- $\beta$ ) or $56^{\circ} \mathrm{C}$ (for Ym1) for 1 minute. Quantification was performed using the comparative threshold cycle method because all the primer pairs (target gene and reference gene) were amplified using comparable efficiencies (relative quantity, $2^{-\Delta \Delta C t}$ ). The replicates were then averaged and fold induction was determined, considering the value at time zero as $1 .^{25}$

\section{Western Blotting}

Tissues from the LV infarcted zone were homogenized in 20 $\mathrm{mmol} / \mathrm{L}$ Tris buffer, $\mathrm{pH} 7.4$, containing $150 \mathrm{mmol} / \mathrm{L}$ sodium chloride, $1 \%$ Triton X-100, and $2 \%$ protease inhibitor cocktail (Sigma-Aldrich). Tissue homogenates were centrifuged and protein concentrations were determined by the Lowry method in the supernatant. ${ }^{26}$ Fifty micrograms of protein from each sample were separated in 12\% SDS-PAGE and electroblotted onto polyvinylidenedifluoride membranes. Blots were blocked with 5\% skimmed milk for 1 hour and incubated overnight at $4^{\circ} \mathrm{C}$ with a polyclonal rabbit $\mathrm{IgG}$ antibody against TGF- $\beta$ (Santa Cruz Biotechnology Inc., Heidelberg, Germany) and against $\beta$-actin (Sigma-Aldrich). After washing with Tris buffer saline and Tween $0.1 \%$, blots were incubated with peroxidase-conjugated secondary antibody (Boster Biological Technology, Pleasanton, CA) for 1.30 hours at room temperature. The specific signals were visualized using the LAS 4100/GE transilluminator (General Electric, Boston, MA). Bands were identified by the use of prestained molecular weight standards (BioRad Laboratories). The relative intensity of the protein signal was quantified by densitometric analysis using the Fluorchem program (Alpha Innotech Corp., San Leandro, CA). Results are expressed as the TGF- $\beta$ protein $/ \beta$-actin protein ratio.
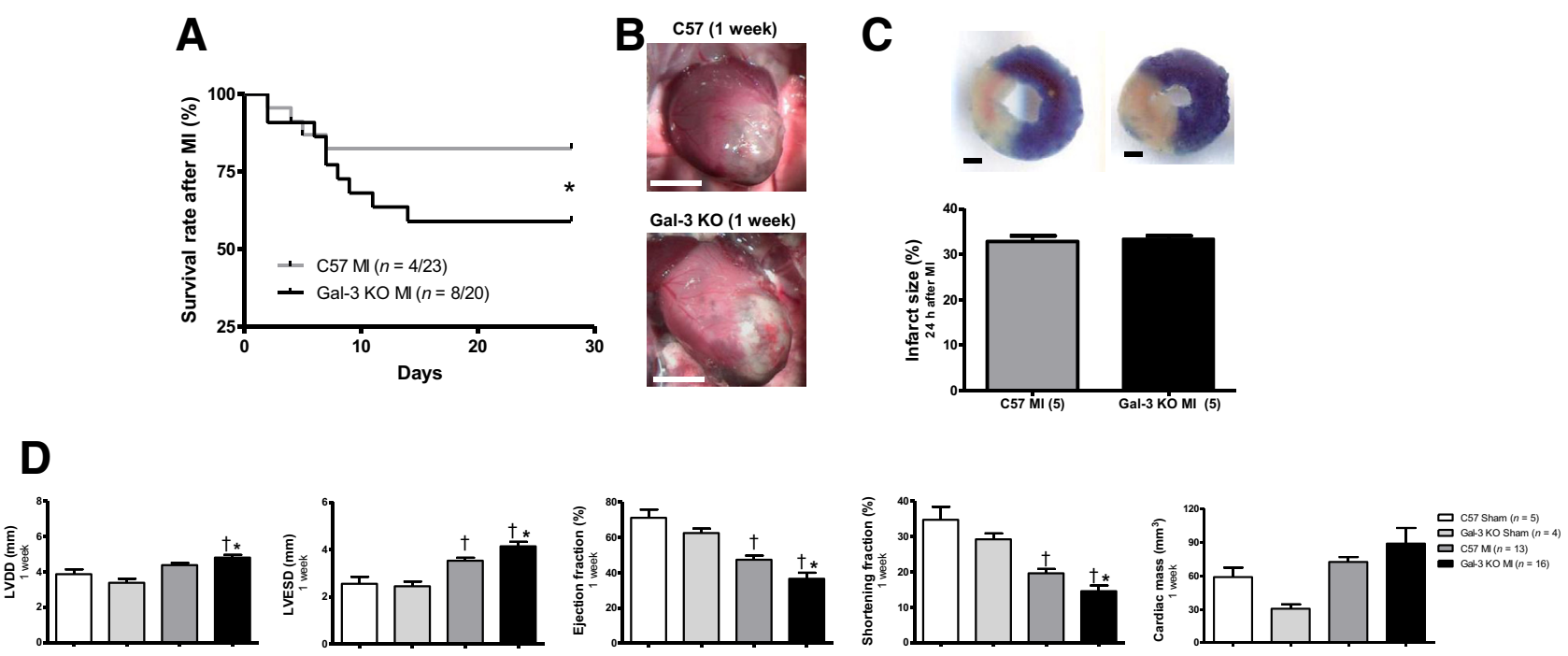

E
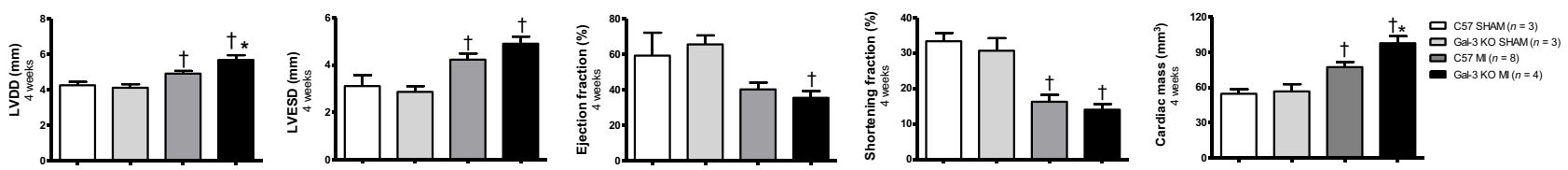

Figure 1 A: Survival rates after myocardial infarction (MI) in C57 and galactin-3 (Gal-3) knockout (K0) (Lgals $3^{-/}$) mice; B: Macroscopy images of hearts with MI. Gal-3 KO mice who died had larger hearts. C: Representative images of Evan's blue and tetrazolium chloride stained section of hearts at 24 hours after MI and infarct size quantification at that time. D and E: Echocardiography in both strains of mice at 1 week (D) and 4 weeks (E). ${ }^{*} P<0.05$ C57 MI versus Gal-3 KO MI mice; ${ }^{\dagger} P<0.05$ versus sham. Scale bars: $5 \mathrm{~mm}$ (B); $1 \mathrm{~mm}$ (C). LVDD, left ventricular end diastolic dimension; LVESD, left ventricular end systolic diameter. 
Table 1 Necropsy at 7 Days and 4 Weeks after MI

\begin{tabular}{|c|c|c|c|c|c|c|c|c|}
\hline \multirow[b]{2}{*}{ Variable } & \multicolumn{4}{|c|}{1 Week after MI } & \multicolumn{4}{|c|}{4 Weeks after MI } \\
\hline & C57 sham & Gal-3 K0 sham & C57 MI & Gal-3 K0 MI & C57 sham & Gal-3 K0 sham & C57 MI & Gal-3 KO MI \\
\hline BW, g & $26 \pm 0.8$ & $28 \pm 0.6$ & $25 \pm 0.4$ & $27 \pm 0.5$ & $30 \pm 1.6$ & $31 \pm 0.5$ & $29 \pm 0.8$ & $31 \pm 0.6$ \\
\hline $\mathrm{HW} / \mathrm{BW}, \mathrm{mg} / \mathrm{g}$ & $5 \pm 0.1$ & $5 \pm 0.2$ & $6 \pm 0.2$ & $6 \pm 0.2^{*}$ & $4 \pm 0.2$ & $4 \pm 0.2$ & $6 \pm 0.2^{\dagger \ddagger}$ & $6 \pm 0.2^{* \S}$ \\
\hline LW/BW, mg/g & $9 \pm 0.4$ & $8 \pm 0.5$ & $9 \pm 0.4$ & $15 \pm 1.2^{*}$ & $5 \pm 0.4$ & $5 \pm 0.1$ & $5 \pm 0.1$ & $6 \pm 0.1$ \\
\hline $\mathrm{HW} / \mathrm{TL}, \mathrm{mg} / \mathrm{g}$ & $7 \pm 0.4$ & $7 \pm 0.4$ & $8 \pm 0.4$ & $9 \pm 0.4^{*}$ & $7 \pm 0.3$ & $8 \pm 0.5$ & $10 \pm 1.4$ & $10 \pm 0.9$ \\
\hline
\end{tabular}

Data are expressed as means \pm SEM.

$* P<0.05$ Gal-3 K0 MI versus Gal-3 K0 sham.

${ }^{\dagger} P<0.05$ C57 MI versus C57 sham.

${ }^{\ddagger} P<0.05$ C57 MI versus 05 Gal-3 K0 sham.

${ }^{\S} P<0.05$ Gal-3 K0 MI versus $C 57$ sham.

${ }^{9} P<0.05$ Gal-3 KO MI versus C57 MI.

BW, body weight; Gal-3, galactin-3, also known as Lgals3; HW, heart weight; K0, knockout; LW, lung weight; MI, myocardial infarction; TL, tibia length.

\section{Statistical Analysis}

Continuous variables are presented as means \pm SEM. Parametric data were analyzed using the unpaired $t$-test to compare the two groups with infarction and one-way analysis of variance, followed by Bonferroni post hoc test for multiple-comparison test using Prism software version 6.0 (GraphPad Software, San Diego, CA). A survival analysis was performed by the Kaplan-Meier method with log-rank test. $P<0.05$ was considered statistically significant.

\section{Results}

\section{Post-MI Survival, Cardiac Remodeling, and Function}

At 4 weeks after MI, the survival rate was significantly reduced in Gal-3 KO mice compared with C57 mice (Figure 1A). After 4 weeks, 4 of 23 C57 mice (17\%) versus 8 of 20 Gal-3 KO mice (40\%) died. On necropsy, dead animals from Gal-3 KO mice had larger hearts (Figure 1B). However, the cause of death was independent of cardiac rupture because $38 \%$ of $\mathrm{C} 57$ and $32 \%$ of Gal-3 KO mice presented with signs of cardiac rupture at necropsy. Infarct size at 24 hours after MI was similar between the groups (Figure 1C).

Echocardiographic examination revealed LV dilation and a significant decrease in contractility at 1 week after MI (Figure 1D). Interestingly, those parameters were even more severely affected in Gal-3 KO mice with MI (Figure 1D), showing that deficiency of Gal-3 unfavorably modified VR and function. LV dilation was still higher in Gal-3 KO mice at 4 weeks (Figure 1D). Long-term LV function was significantly reduced in both genotypes with MI (Figure 1D). At 4 weeks, although systolic function in MI groups was similar, the dysfunction in Gal-3 KO mice after MI started earlier in the evolution of MI (Figure 1, D and E).

\section{Necropsy}

By the end of the first week, cardiac mass index and the lung weight/body weight ratio (pulmonary congestion) significantly increased in Gal-3 KO mice with MI compared with the sham and MI controls, suggesting a deficiency of Gal-3 in animals with MI exacerbated cardiac remodeling and failure (Table 1). To determine whether lack of Gal-3 affected the size of the infarction, the injury zone was also measured at 1 day after MI. The infarct size at 24 hours was $33 \% \pm 2.2 \%$ in $\mathrm{C} 57$ mice and $33 \% \pm 0.5 \%$ in Gal-3 KO mice. During the chronic phase, ventricular hypertrophy after MI was significantly higher in both groups, whereas pulmonary congestion remained compensated (Table 1).

\section{Macrophage Infiltration and Expression of Markers of Alternative Activation at 7 Days after MI}

Deletion of Gal-3 markedly prevented macrophage infiltration at the infarct zone (Figure 2A) and significantly increased the expression of mannose receptor (CD206) and YM1 (both markers of M2 macrophages), suggesting that Gal-3 is not only essential for inducing early macrophage infiltration but also for a proinflammatory M1 profile (Figure 2, B and C).

\section{Cytokine Expression}

The infarct zone of C57 mice exhibited considerable high amounts of mRNA for TNF- $\alpha$, whereas in Gal-3 KO mice it was completely prevented (Figure 3A). In contrast, the realtime quantitative RT-PCR analysis found that the expression of IL-10 and IL-6 was higher in the infarct zone of Gal-3 KO mice than in C57 mice (Figure 3, B and C).

\section{Collagen Concentration, MMP-2 Activity, and TGF- $\beta$ Expression}

Lack of Gal-3 in mice with MI markedly prevented the increase in collagen at the infarct zone throughout the MI evolution (Figure 4A). In addition, at 1 week after MI, the gelatinolytic activity of MMP-2 was highly enhanced in Gal-3 KO mice (Figure 4B). Moreover, lack of Gal-3 prevented the expression of TGF- $\beta$ (Figure $4 \mathrm{C}$ ). Thus, these 

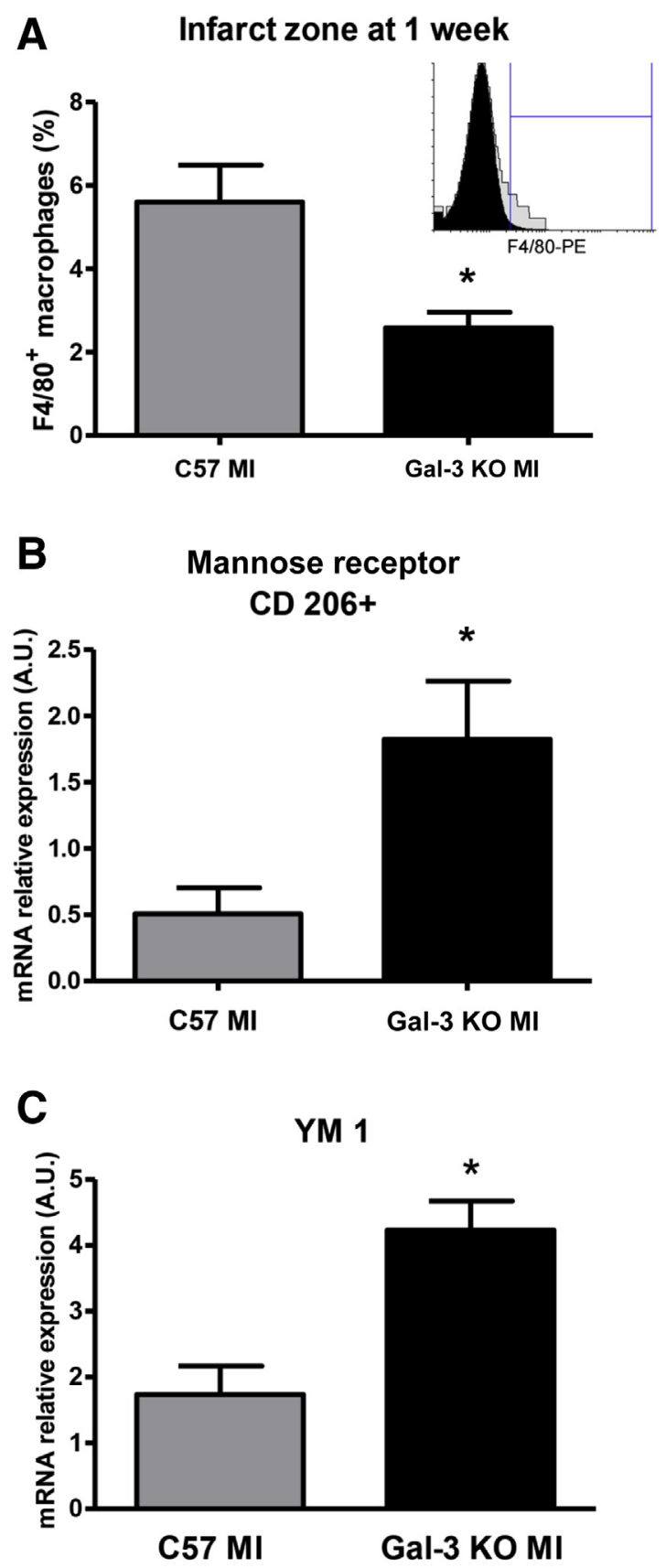

Figure 2 A: Quantification of $\mathrm{F}_{4} / 80^{+}$macrophages. Inset: Overlay histogram showing $\mathrm{C} 57$ myocardial infarction (MI) samples in gray and galactin-3 (Gal-3) knockout (KO) ( $\left.\mathrm{ggals}^{-/-}\right)$MI samples in black. The blue marker of the inset shows specific staining of the indicated antibody. B and C: Expression of M2 phenotype markers CD206+ (also known as Mrc1) and YM1 (also known as Chil1) in the infarct zone at 1 week. $n=5$ C57 MI (A); $n=6$ Gal-3 KO MI (A); $n=3$ C57 MI (B and C); $n=8$ Gal-3 KO MI (B and $\mathbf{C}) .{ }^{*} P<0.05$ versus C57. A.U., arbitrary units.

results indicate that Gal-3 regulates not only the synthesis of collagen but also the activity of MMP-2.

At an early stage, myocardial fibrosis at the remote zone was similar among groups (Figure 5A), whereas the progressive increase in fibrosis observed in C57 MI mice at 4 weeks was prevented in Gal-3 KO mice (Figure 5B).
Histologic and Morphometric Analysis

Scar thickness and scar thickness ratio were quantified at 1 and 4 weeks (Figure 6, A and B). At 1 week after MI, the scar thickness was significantly reduced in Gal-3 KO mice
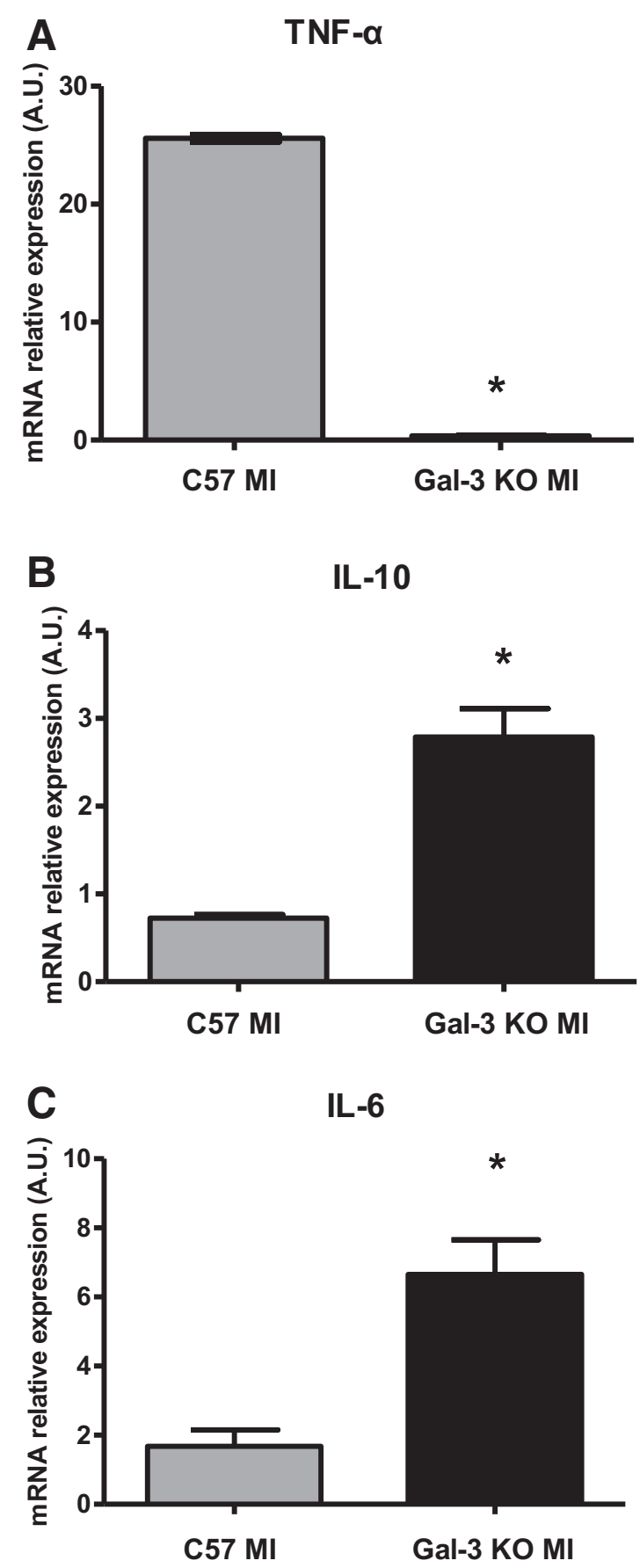

Figure 3 Quantification of cytokine expression by quantitative realtime RT-PCR (RT-qPCR) in the infarct zone at 7 days. A: The lack of galactin-3 (Gal-3) significantly prevents the expression of tumor necrosis factor Tnf (also known as TNF- $\alpha$ ). B and C: At the same time, the lack of Gal3 further enhances the expression of Il10 (also known as IL-10) (B) and Il6 (also known as IL-6) (C). $n=3$ C57 MI (A-C); $n=8$ Gal-3 KO MI (A-C) ${ }^{*} P<0.05$ versus C57. A.U., arbitrary units; Gal-3, galactin-3, also known as Lgals3; K0, knockout; MI, myocardial infarction. 

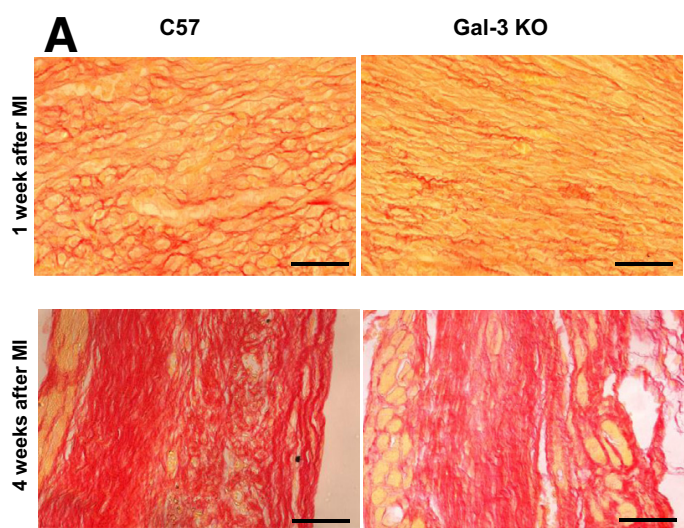

B

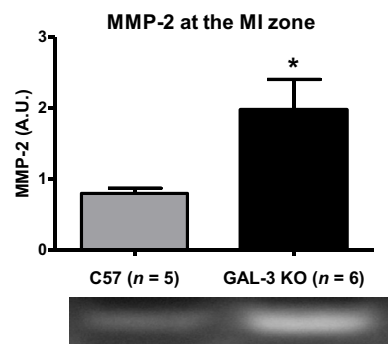

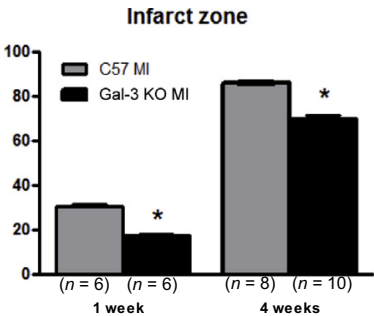

C

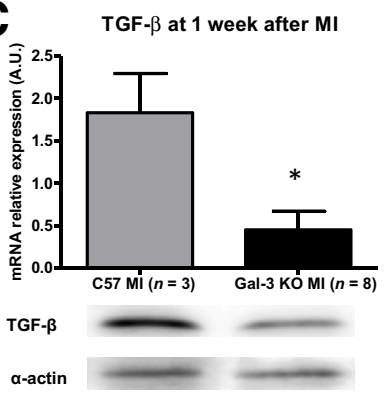

Figure 4 A: Genetic deletion of galactin-3 (Gal-3, also known as Lgals3), extensively prevents the collagen deposition in the myocardial infarct (MI) region at 1 and 4 weeks. B: Representative image of zymographs and quantification of matrix metalloproteinase (MMP)-2 activity in the infarct region at 1 week after myocardial infarction (MI). C: Genetic deletion of Gal-3 significantly attenuates the expression of transforming growth factor (Tgfb1, also known as TGF- $\beta$ ). Number of animals is shown between parentheses. ${ }^{*} P<0.05$ versus $\mathrm{C} 57$ mice. Original magnification, $\times 400(A)$. Scale bars $=40 \mu \mathrm{m}(\mathrm{A})$. A.U., arbitrary units; K0, knockout. compared with C57. Although at 4 weeks, no differences were found between the groups, in C57 mice the scar thickness was progressively decreasing from 1 to 4 weeks, whereas in Gal-3 KO mice the scar thickness was thinnest from the early stage of MI (Figure 6, A and B). At 1 and 4 weeks, the infarct size was significantly larger in Gal-3 KO animals but similar at 4 weeks (Figure $6 \mathrm{C}$ ). With all results considered together, the discrepancy observed between
A

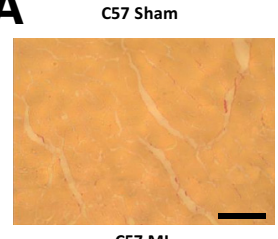

C57 MI

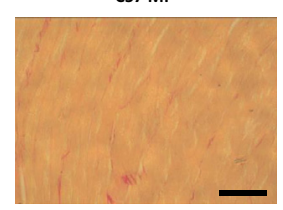

B

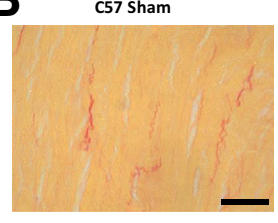

C57 MI

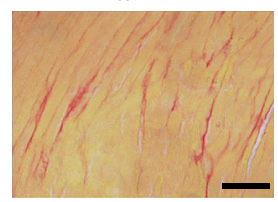

Gal-3 ko Sham

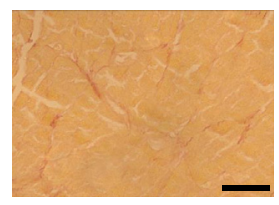

Gal-3 Ko MI

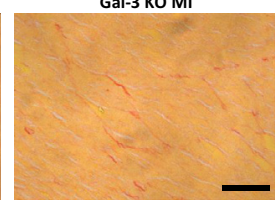

Gal-3 Ko Sham

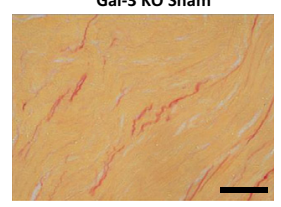

Gal-3 Ko MI

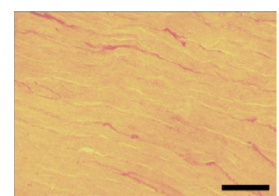

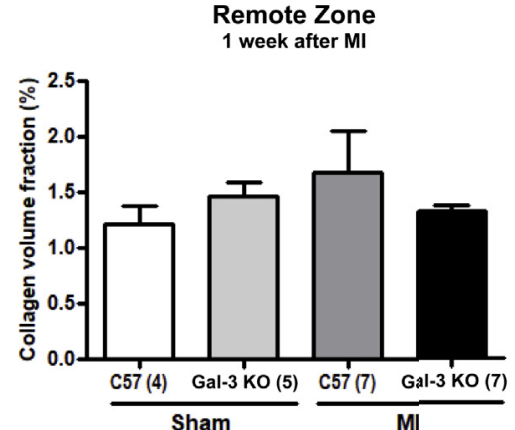

Remote Zone 4 weeks after MI

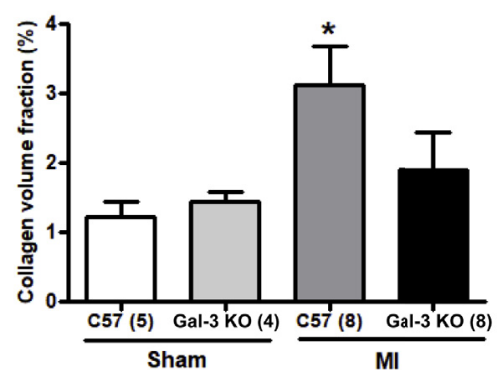

Figure 5 Myocardial collagen volume fraction quantification at remote zone at 1 week (A) and 4 weeks (B) after myocardial infarction (MI) or sham operation. No differences are observed after 1 week between the sham and MI groups. MI significantly increases the fibrosis at remote zone after 4 weeks, which is extensively prevented in galactin-3 (Gal-3) knockout (KO) ( ggals3 $^{-/-}$) mice. The number of mice analyzed per group are in parentheses on the image. ${ }^{*} P<0.05$ versus Gal-3 KO MI. Scale bars $=40 \mu \mathrm{m}$ (A and B). 

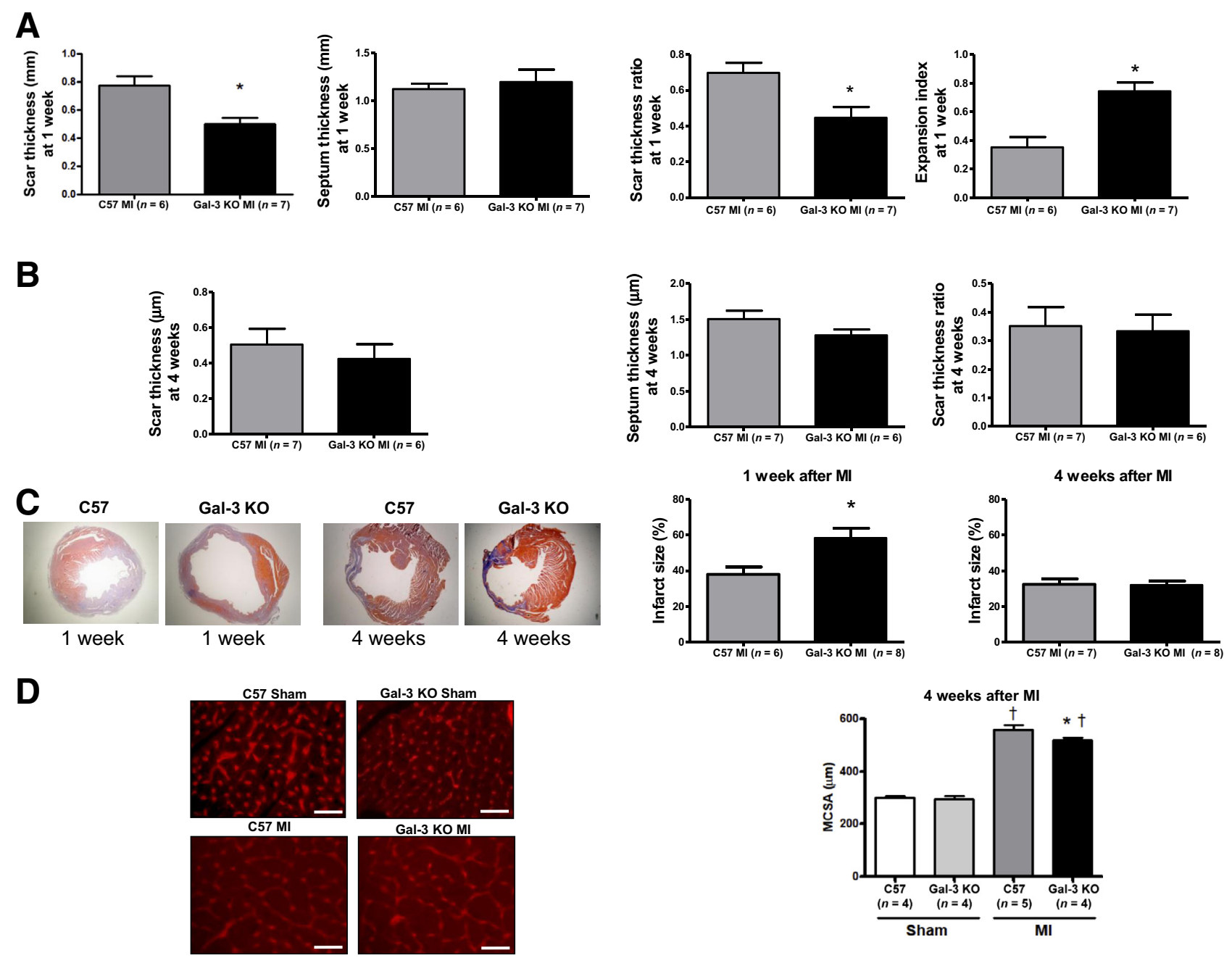

Figure 6 A: At 1 week, galactin-3 (Gal-3) knockout (K0) mice, also known as Lgals $3^{\%-}$ mice, with myocardial infarction (MI) have the thinnest infarct zone, larger expansion, and larger ventricular cavity size. B: At 4 weeks after MI, scar and septum thicknesses are similar between the groups. C: Representative images of Masson trichrome stained sections and infarct size at 1 and 4 weeks. D: Representative images of rodhamine staining section and quantification of myocyte crosssectional area (MCSA) in the remote zone at 4 weeks. MI progresively increases MCSA from the early stages; at 4 weeks, MCSA is slightly but significantly attenuated in infarcted Gal-3 K0 mice. ${ }^{*} P<0.05$ versus C57 MI mice; ${ }^{\dagger} P<0.05$ versus mice in the sham group. Original magnification: $\times 20$ (C); $\times 400$ (D). Scale bars $=40$ $\mu \mathrm{m}$ (D).

infarct size at 24 hours, 7 days, and 4 weeks suggests that larger MI observed at 7 days in Gal-3 KO mice may be related to larger expansion but not necessarily to major infarct size. Myocyte cross-sectional area at 4 weeks was significantly increased in both strains with MI. However, the progressive increase in hypertrophy observed in C57 mice with MI was clearly prevented in mice that lacked Gal-3 (Figure 6D).

\section{Discussion}

The temporal evolution of wound healing after MI begins with a robust inflammatory response followed by a progressive suppression of inflammation and the formation of a fibrous scar. Gal-3 plays an essential role in cardiovascular pathophysiologic processes by regulating acute and chronic inflammatory responses. ${ }^{16,18,27-30}$ However, the role of that lectin in the temporal evolution of wound healing, VR, and function after MI is still far from being elucidated. We found that Gal-3 regulates macrophage infiltration from the onset of MI as well as the expression of macrophage markers for alternative activation and the expression of proinflammatory and anti-inflammatory cytokines. Interestingly, the lack of Gal-3 significantly reduced the survival. In addition, these findings corroborate that Gal-3 is a key mediator for the formation and remodeling of the fibrous scar because the lack of Gal-3 attenuated the expression of TGF- $\beta$ and increased the activity of MMP-2 in the infarct zone, prevented the collagen deposition along the evolution of MI, and increased the scar thickness ratio and expansion index. Finally, lack of Gal-3 severely exacerbated the evolution of global remodeling and dysfunction from the beginning of MI. Thus, these findings strongly suggest that Gal-3 is a determinant factor for 
the early stage of healing and remodeling after MI. These results also indicate that the leading role of Gal-3 is not only limited to the infarct zone wound healing but also has a direct influence on cardiac structural changes and function along the temporal evolution of MI.

A few hours after the loss of cardiac myocytes, necrotic cells are removed by an intense and coordinated immune response that completes the replacement of the injured area with scar tissue. The development of this immune response has direct implications in the evolution of VR. After MI, a complex pool of signals from myocardial injury induces macrophage infiltration and differentiation. Gal-3 increases early in the injured zone and reaches the maximum expression from 7 to 14 days afrer MI. ${ }^{17,19}$ Previous studies suggested that Gal-3 is required for macrophage infiltration and function, cytokine expression, and collagen synthesis in cardiovascular pathology. ${ }^{16,27-29}$ However, the role of Gal3 in the temporal evolution of wound healing and its involvement in the evolution of $\mathrm{RV}$ are unknown. We analyzed the role of Gal-3 in the evolution of macrophage infiltration and its influence on VR and function at 1 and 4 weeks after MI. At 1 week after MI, we found that Gal-3 deficiency reduces the infiltration of macrophages and the expression of TNF- $\alpha$ and increases the expression of M2 macrophage markers, IL-6, and IL-10. Recently, Shirakawa et $\mathrm{al}^{31}$ reported that Gal-3 is markedly increased in macrophages isolated from MI and contributes to their phenotypic polarization and repairing, at least in part by secreting proinflammatory and anti-inflammatory cytokines. In hypertension, Gal-3 KO mice enhanced the expression of IL$10,{ }^{16}$ and this cytokine promoted the infiltration of M2 macrophages in the MI area. ${ }^{31}$ Thus, these results, in line with previous reports, ${ }^{16,31}$ add new information and indicate that Gal-3 is a critical player in the regulation of temporal evolution of healing, scar formation, and global remodeling and function. These data strongly suggest that Gal-3 is required for the temporal evolution of phenotypic and functional polarization of M1/M2 macrophages and proinflammatory and anti-inflammatory cytokine expression in wound healing. Surprisingly, under the experimental conditions of this study, Gal-3 KO mice with infarction had a greater increase in IL-6; however, why the deficit of Gal-3 increased IL-6 is not completely clear. It is generally accepted that IL-6 has proinflammatory and profibrotic functions, ${ }^{32}$ and this study found that the lack of Gal-3 leads to a reduction in IL- $6 .{ }^{16}$ Nevertheless, ambivalent effects of IL-6 have been described to rely on the activation of two major signaling cascades. ${ }^{33}$ Accordingly, under certain experimental conditions, the deficit of Gal-3 was linked to an increase in IL- $6 .^{34,35}$ Because IL-10 and IL-6 have recently been reported to promote the polarization of M2 macrophages through STAT3, ${ }^{31}$ the current study results strongly suggest that after MI both cytokines may contribute to the mechanism by which Gal-3 regulates the enhanced expression of markers clearly indicative of macrophage polarization toward the M2 subtype.
The reduction in collagen synthesis by Gal-3 deficiency has been widely established. ${ }^{16,27,28,36}$ After MI, the synthesis of collagen in the infarct zone starts within the first 7 days and progressively reaches a plateau between 4 and 6 weeks later. ${ }^{37}$ The dynamics of collagen synthesis is an important determinant of the mechanical properties and remodeling of the infarcted wall and contributes to the evolution of the global VR. Thus, the decrease in fibrosis reduces the tensile strength of the infarcted wall, favoring the expansion and adverse remodeling. ${ }^{22}$ These findings confirm that the deficit of Gal-3 prevents collagen synthesis in the MI area and the remote zone throughout the evolution of MI.

TGF- $\beta$ is upregulated after MI, and its signaling pathways actively participate in post-MI healing and remodeling. ${ }^{38}$ After MI, macrophages and lymphocytes that infiltrate into the infarct zone are known to release myocardial TGF- $\beta$ for promoting fibroblast activation and collagen synthesis. ${ }^{39}$ Studies performed in culture fibroblasts found that TGF- $\beta$ leads to extracellular protein synthesis and concomitant suppression of MMP. ${ }^{40}$ In the present study, the increase in TGF- $\beta$ observed in the infarct zone of control animals was markedly attenuated in Gal-3 KO mice, suggesting a reduction in collagen synthesis. TGF- $\beta$ is a key molecular link between the inflammatory and reparative response. However, the cellular response to TGF- $\beta$ at early stages after MI may be attenuated by the abundance of other proinflammatory mediators. When debris from necrotic tissue is cleared, TGF- $\beta$ in association with other neurohormonal mediators and Gal-3 regulate fibroblast proliferation and fibrogenic response. Nevertheless, although the reduction in fibrosis observed in Gal-3 KO mice may be explained by a reduction in TGF- $\beta$, these results also indicate that it is not the only mechanism because the lower collagen amount observed in the MI zone of KO mice could also be the result of an increase in the collagenolytic activity of MMP-2. The enhancement of MMP-2 activity seems to be important in wound healing and remodeling of the infarct zone because the MMP-2 deletion or pharmacologic inhibition reduces both cardiac rupture and remodeling after MI in mice. ${ }^{41}$ Previous findings identified that Gal-3 is a substrate that gelatinizes MMP-2 and that its degradation may play a role in modulating the biological activities of Gal-3, ${ }^{42}$ which may explain the enhancement in the activity of MMP-2 found in Gal-3 KO mice with MI. Thus, these results indicate that in the evolution of the reparative process, Gal-3 plays a critical role not only in the synthesis but also in the collagen turnover in the MI area.

Ben-Mordechai et $\mathrm{al}^{43}$ reported that a depletion of macrophages markedly increases post-MI mortality in mice and that mesenchymal stem cells could influence post-MI healing in part by modulating the macrophage phenotype. Therefore, the anti-inflammatory and antifibrotic effects observed by depletion of Gal-3 from the beginning of MI strongly suggest that the greater expansion, adverse remodeling, and lower survival observed in those mice could have occurred because of the factors 
mentioned above. In these experiments, the survival rate in Gal-3 KO mice with MI was significantly reduced. The cause of death was independent of cardiac rupture but closely related to the effect of the lack of Gal-3 on healing and remodeling. Necropsy of the dead animals found cardiac rupture in $38 \%$ of the C57 mice and 32\% of the Gal-3 KO mice. Although other causes of mortality were not studied here, the adverse early remodeling and pulmonary congestion observed in Gal-3 KO mice with MI strongly suggest that uncompensated heart failure may be the main cause of death. Furthermore, the possibility of arrhythmias should also be considered.

The dynamics of the infarct zone healing are closely related to the natural evolution of VR. This remodeling is characterized by the progressive thinning of the MI area followed by hypertrophy and fibrosis of the noninfarcted myocardium, ventricular dilation, and failure. The benefits or the counterproductive effects of inhibiting the reparative process in the evolution of the RV is a matter of controversy. ${ }^{6}$ Studies in animals reported that the inhibition of the reparative process can reduce the infarct size and improve the evolution of the VR. ${ }^{44,45}$ However, the early use of anti-inflammatory drugs after infarction promoted the thinning and expansion of the infarct zone in animals and increased the incidence of cardiac rupture in patients. ${ }^{46}$ Thus, a defect in the healing process or an increase in the infarct size and parietal stress were the main determinants of the expansion and the appearance of adverse clinical events, heart failure, and death. ${ }^{22}$ In line with the affected natural evolution of the reparative process, we found that Gal-3 KO mice with MI exhibited a reduction in the scar thickness ratio, enhanced infarct expansion, and ventricular dilation and dysfunction. The expansion of the MI zone refers to the radial thinning and circumferential increase in the extent of the infarct area that occurs during the days to weeks after an acute MI. Previous reports found different pathologic mechanisms that explain the link between the inflammatory process during healing and its relationship with the global VR and dysfunction. ${ }^{16,22,47}$ In this regard, it was previously found that the reduction in the inflammatory cellular infiltrate delays the reparative response after MI and increases scar thinning and expansion, dilation, and systolic dysfunction. ${ }^{22}$ These findings indicate that at 7 days, Gal-3 KO mice had the significantly thinnest scar and larger expansion when compared with controls. These results aid our understanding, at least in part, of the discrepancy observed between the infarct size at 7 days when compared with 1 day and 4 weeks. The method used for measuring the scar size is based on the quantification of the internal and external length of the infarcted segment in relation to the total perimeter of the ventricular cavity. Because early stages of post-MI remodeling are mainly limited to infarct region and accompanied by a thinner and larger expansion, this finding may explain the reason why Gal-3 KO mice had larger infarct sizes than C57 mice at 1 week after MI but similar infarct sizes at 1 day and 4 weeks. Furthermore, a natural positive selection of the healthiest animals may explain the discrepancy that we observed in the evolution of the remodeling and mortality that allowed mice with MI to survive for 4 weeks.

Despite the potential role of $\mathrm{Gal}-3$ as a prognostic biomarker in heart failure, ${ }^{18}$ at 4 weeks after MI, differences in cardiac hypertrophy (heart weight/body weight and heart weight/tibia length) or cardiac function (LV ejection fraction and fractional shortening) were not found, whereas differences were still found in the collagen content and myocyte cross-sectional area between both genotypes. A recent study found that Gal-3 is produced from macrophages and myofibroblasts at the early phase after pressure overload and from cardiomyocytes at the late phase. ${ }^{48}$ This investigation did not study whether Gal-3 is increased in cardiomyocyte at chronic remodeling. However, on the basis of those results, although the lack of differences may still be attributable to the natural selection of the healthiest animals that survived to 4 weeks, the role of Gal-3 on late phase of remodeling seems to be less relevant when compared with the early phase. Nevertheless, the loss of significance in cardiac function and remodeling at 4 weeks should be interpreted with caution because the temporal evolution from 1 to 4 weeks reveals that genetic mutation of Gal-3 KO mice accelerated the adverse remodeling. Therefore, our findings strongly suggest that Gal-3 is a key player required for the temporal evolution of alternative macrophages, cytokine expression, collagen turnover, and scar formation during the wound healing process, which plays a crucial role in structural remodeling, and function.

In summary, Gal-3 is an essential regulatory factor of postMI healing because it regulates the dynamics of the reparative process, the phenotypic profile of macrophages, the proinflammatory and anti-inflammatory cytokine expression, and fibrosis with the temporal evolution of the healing process in mice. The deficit of Gal-3 diminished macrophage infiltration, thus altering its phenotypic polarization and consequently the dynamics of the reparative process, increasing the expansion and aggravating the functional and histomorphometric evolution of cardiac remodeling. Our results strengthen the theory that Gal-3 is essential for early wound healing. The fact that the lack of Gal-3 prevented the 4-week fibrosis at the remote zone suggests that blocking Gal-3 after healing while allowing its physiologic response in the early phase may have a beneficial effect on chronic remodeling. Additional studies on clarifying the role of blocking Gal-3 on long-term remodeling are needed.

\section{Study Limitations}

The role of TGF- $\beta$ has been widely studied in experimental models of MI and remodeling. ${ }^{39,49}$ Our results indicate that the lack of Gal-3 prevents the increase of TGF- $\beta$ expression. However, because we used an in vivo model of MI, these results are limited to understanding the attenuated expression of TGF- $\beta$ by Gal-3 KO at the infarct zone. Additional studies examining the molecular basis involved in Gal3 -dependent TGF- $\beta$ expression are required. A deep understanding of the role of Gal-3 in the regulation of this 
multifunctional growth factor in healing and remodeling requires performing studies in vitro, which the current study protocol was not originally designed to do. Thus, further investigation of the translational relevance of the present study is needed.

\section{Acknowledgments}

We thank Ana Chiaro for technical assistance and Sergio Mazzini for reviewing this manuscript.

\section{References}

1. Opie LH, Commerford PJ, Gersh BJ, Pfeffer MA: Controversies in ventricular remodelling. Lancet 2006, 367:356-367

2. Frangogiannis NG: The inflammatory response in myocardial injury, repair, and remodelling. Nat Rev Cardiol 2014, 11:255-265

3. Clarke SA, Richardson WJ, Holmes JW: Modifying the mechanics of healing infarcts: is better the enemy of good? J Mol Cell Cardiol 2016, 93:115-124

4. Weinberger T, Schulz C: Myocardial infarction: a critical role of macrophages in cardiac remodeling. Front Physiol 2015, 6:107

5. Chen B, Frangogiannis NG: Immune cells in repair of the infarcted myocardium. Microcirculation 2017, 24:1-10

6. Anzai T: Post-infarction inflammation and left ventricular remodeling: a double-edged sword. Circ J 2013, 77:580-587

7. Wan E, Yeap XY, Dehn S, Terry R, Novak M, Zhang S, Iwata S, Han X, Homma S, Drosatos K, Lomasney J, Engman DM, Miller SD, Vaughan DE, Morrow JP, Kishore R, Thorp EB: Enhanced efferocytosis of apoptotic cardiomyocytes through myeloid-epithelialreproductive tyrosine kinase links acute inflammation resolution to cardiac repair after infarction. Circ Res 2013, 113:1004-1012

8. DeBerge M, Yeap XY, Dehn S, Zhang S, Grigoryeva L, Misener S, Procissi D, Zhou X, Lee DC, Muller WA, Luo X, Rothlin C, Tabas I, Thorp EB: MerTK cleavage on resident cardiac macrophages compromises repair after myocardial ischemia reperfusion injury. Circ Res 2017, 121:930-940

9. Horckmans M, Ring L, Duchene J, Santovito D, Schloss MJ, Drechsler M, Weber C, Soehnlein O, Steffens S: Neutrophils orchestrate post-myocardial infarction healing by polarizing macrophages towards a reparative phenotype. Eur Heart J 2017, 38:187-197

10. Das A, Sinha M, Datta S, Abas M, Chaffee S, Sen CK, Roy S: Monocyte and macrophage plasticity in tissue repair and regeneration. Am J Pathol 2015, 185:2596-2606

11. Koh TJ, DiPietro LA: Inflammation and wound healing: the role of the macrophage. Expert Rev Mol Med 2011, 13:e23

12. Mantovani A, Sica A, Sozzani S, Allavena P, Vecchi A, Locati M: The chemokine system in diverse forms of macrophage activation and polarization. Trends Immunol 2004, 25:677-686

13. Wynn TA, Vannella KM: Macrophages in tissue repair, regeneration, and fibrosis. Immunity 2016, 44:450-462

14. Panizzi P, Swirski FK, Figueiredo JL, Waterman P, Sosnovik DE, Aikawa E, Libby P, Pittet M, Weissleder R, Nahrendorf M: Impaired infarct healing in atherosclerotic mice with Ly-6Chi monocytosis. J Am Coll Cardiol 2010, 55:1629-1638

15. Dutta $\mathrm{P}$, Nahrendorf M: Monocytes in myocardial infarction. Arterioscler Thromb Vasc Biol 2015, 35:1066-1070

16. González GE, D’Ambrosio MA, Nakagawa P, Liao TD, Peterson EL, Leung P, Dai X, Janic B, Liu YH, Yang XP, Carretero OA: RNE: cardiac-deleterious role of galectin-3 in chronic angiotensin IIinduced hypertension. Am J Physiol Heart Circ Physiol 2016, 311: H1287-H1296
17. Sanchez-Mas J, Lax A, Asensio-Lopez MC, Fernandez-Del Palacio MJ, Caballero L, Garrido IP, Pastor F, Januzzi JL, PascualFigal DA: Galectin-3 expression in cardiac remodeling after myocardial infarction. Int J Cardiol 2014, 172:e98-e101

18. de Boer RA, Lok DJA, Jaarsma T, van der Meer P, Voors AA, Hillege HL, van Veldhuisen DJ: Predictive value of plasma galectin-3 levels in heart failure with reduced and preserved ejection fraction. Ann Med 2011, 43:60-68

19. Mosleh W, Chaudhari MR, Sonkawade S, Mahajan S, Khalil C, Frodey K, Shah T, Dahal S, Karki R, Katkar R, Blankesteijn WM, Page B, Pokharel S, Kim M, Sharma UC: The therapeutic potential of blocking galectin-3 expression in acute myocardial infarction and mitigating inflammation of infarct region: a clinical outcome-based translational study. Biomarker Insights 2018, 13. 1177271918 771969

20. González GE, Cassaglia P, Noli Truant S, Fernández MM, Wilensky L, Volberg V, Malchiodi EL, Morales C, Gelpi RJ: Galectin-3 is essential for early wound healing and ventricular remodeling after myocardial infarction in mice. Int J Cardiol 2014, 176:1423-1425

21. Committee for the Update of the Guide for the Care and Use of Laboratory Animals; National Research Council: Guide for the Care and Use of Laboratory Animals: Eighth Edition. Washington, DC, National Academies Press, 2011

22. González GE, Seropian IM, Krieger ML, Palleiro J, Lopez Verrilli MA, Gironacci MM, Cavallero S, Wilensky L, Tomasi VH, Gelpi RJ, Morales C: Effect of early versus late AT ${ }_{1}$ receptor blockade with losartan on postmyocardial infarction ventricular remodeling in rabbits. Am J Physiol Heart Circ Physiol 2009, 297 : H375-H386

23. Dai W, Wold LE, Dow JS, Kloner RA: Thickening of the infarcted wall by collagen injection improves left ventricular function in rats: a novel approach to preserve cardiac function after myocardial infarction. J Am Coll Cardiol 2005, 46:714-719

24. Seropian IM, Toldo S, Van Tassell BW, Abbate A: Anti-inflammatory strategies for ventricular remodeling following ST-segment elevation acute myocardial infarction. J Am Coll Cardiol 2014, 63: $1593-1603$

25. Schmittgen TD, Livak KJ: Analyzing real-time PCR data by the comparative CT method. Nat Protoc 2008, 3:1101-1108

26. Lowry OH, Rosebrough NJ, Farr AL, Randall RJ: Protein measurement with the Folin phenol reagent. J Biol Chem 1951, 193:265-275

27. Sharma UC, Pokharel S, van Brakel TJ, van Berlo JH, Cleutjens JPM, Schroen B, André S, Crijns HJGM, Gabius H-J, Maessen J, Pinto YM: Galectin-3 marks activated macrophages in failure-prone hypertrophied hearts and contributes to cardiac dysfunction. Circulation 2004, 110:3121-3128

28. Liu YH, D'Ambrosio M, Liao T-D, Peng H, Rhaleb N-E, Sharma U, Andre S, Gabius HJ, Carretero OA: N-acetyl-seryl-aspartyl-lysylproline prevents cardiac remodeling and dysfunction induced by galectin-3, a mammalian adhesion/growth-regulatory lectin. Am J Physiol Heart Circ Physiol 2009, 296:H404-H412

29. Solano de Freitas Souza B, Nascimento Silva D, Hughes Carvalho R, Louise de Almeida Sampaio G, Diaz Paredes B, Aragão França L, Machado Azevedo C, Fraga Vasconcelos J, Santana Meira C, Chenaud Neto P, Garcia Macambira S, da Silva KN, James Allahdadi K, Tavora F, David de Souza Neto J, Ribeiro dos Santos R, Botelho Pereira Soares M, de Pesquisas Gonçalo Moniz C, Oswaldo Cruz F, Waldemar R: Association of cardiac galectin-3 expression, myocarditis, and fibrosis in chronic chagas disease cardiomyopathy. Am J Pathol 2017, 187:1134-1146

30. Meijers WC, van der Velde AR, Pascual-Figal DA, de Boer RA: Galectin-3 and post-myocardial infarction cardiac remodeling. Eur J Pharmacol 2015, 763:115-121

31. Shirakawa K, Endo J, Kataoka M, Katsumata Y, Yoshida N, Yamamoto T, Isobe S, Moriyama H, Goto S, Kitakata H, Hiraide T, Fukuda K, Sano M: IL-10-STAT3-Galectin-3 axis is essential for 
osteopontin-producing reparative macrophage polarization after myocardial infarction. Circulation 2018, 138:2021-2035

32. Gonzalez GE, Rhaleb NE, D'Ambrosio MA, Nakagawa P, Liu Y, Leung P, Dai X, Yang XP, Peterson EL, Carretero OA: Deletion of interleukin-6 prevents cardiac inflammation, fibrosis and dysfunction without affecting blood pressure in angiotensin II-high salt-induced hypertension. J Hypertens 2015, 33:144-152

33. Scheller J, Chalaris A, Schmidt-Arras D, Rose-John S: The pro- and anti-inflammatory properties of the cytokine interleukin-6. Biochim Biophys Acta 2011, 1813:878-888

34. Farnworth SL, Henderson NC, MacKinnon AC, Atkinson KM, Wilkinson T, Dhaliwal K, Hayashi K, Simpson AJ, Rossi AG, Haslett C, Sethi T: Galectin-3 reduces the severity of pneumococcal pneumonia by augmenting neutrophil function. Am J Pathol 2008, 172:395-405

35. Wu S-Y, Yu J-S, Liu F-T, Miaw S-C, Wu-Hsieh BA: Galectin-3 negatively regulates dendritic cell production of IL-23/IL-17-axis cytokines in infection by histoplasma capsulatum. J Immunol 2013, 190:3427-3437

36. Yu L, Ruifrok WPT, Meissner M, Bos EM, Van Goor H, Sanjabi B, Van Der Harst P, Pitt B, Goldstein IJ, Koerts JA, Van Veldhuisen DJ, Bank RA, Van Gilst WH, Silljé HHW, De Boer RA: Genetic and pharmacological inhibition of galectin-3 prevents cardiac remodeling by interfering with myocardial fibrogenesis. Circ Hear Fail 2013, 6: $107-117$

37. Morales C, González GE, Rodríguez M, Bertolasi CA, Gelpi RJ: Histopathologic time course of myocardial infarct in rabbit hearts. Cardiovasc Pathol 2002, 11:339-345

38. Vilahur G, Juan-Babot O, Peña E, Oñate B, Casaní L, Badimon L: Molecular and cellular mechanisms involved in cardiac remodeling after acute myocardial infarction. J Mol Cell Cardiol 2011, 50:522-533

39. Frangogiannis NG: The extracellular matrix in ischemic and nonischemic heart failure. Circ Res 2019, 125:117-146

40. Leask A: Targeting the TGF $\beta$, endothelin-1 and CCN2 axis to combat fibrosis in scleroderma. Cell Signal 2008, 20:1409-1414
41. Matsumura SI, Iwanaga S, Mochizuki S, Okamoto H, Ogawa S, Okada Y: Targeted deletion or pharmacological inhibition of MMP-2 prevents cardiac rupture after myocardial infarction in mice. J Clin Invest 2005, 115:599-609

42. DeCoux A, Lindsey ML, Villarreal F, Garcia RA, Schulz R: Myocardial matrix metalloproteinase-2: inside out and upside down. J Mol Cell Cardiol 2014, 77:64-72

43. Ben-Mordechai T, Holbova R, Landa-Rouben N, Harel-Adar T, Feinberg MS, Abd Elrahman I, Blum G, Epstein FH, Silman Z, Cohen S, Leor J: Macrophage subpopulations are essential for infarct repair with and without stem cell therapy. J Am Coll Cardiol 2013, 62:1890-1901

44. Jiang Y, Bai J, Tang L, Zhang P, Pu J: Anti-CCL21 antibody attenuates infarct size and improves cardiac remodeling after myocardial infarction. Cell Physiol Biochem 2015, 37:979-990

45. Jung M, Ma Y, Iyer RP, DeLeon-Pennell KY, Yabluchanskiy A, Garrett MR, Lindsey ML: IL-10 improves cardiac remodeling after myocardial infarction by stimulating M2 macrophage polarization and fibroblast activation. Basic Res Cardiol 2017, 112:33

46. Silverman HS, Pfeifer MP: Relation between use of antiinflammatory agents and left ventricular free wall rupture during acute myocardial infarction. Am J Cardiol 1987, 59:363-364

47. Seropian IM, Cerliani JP, Toldo S, Van Tassell BW, Ilarregui JM, Gonzalez GE, Matoso M, Salloum FN, Melchior R, Gelpi RJ, Stupirski JC, Benatar A, Gomez KA, Morales C, Abbate A, Rabinovich GA: Galectin-1 controls cardiac inflammation and ventricular remodeling during acute myocardial infarction. Am J Pathol 2013, 182:29-40

48. Meijers WC, López-Andrés N, de Boer RA: Galectin-3, cardiac function, and fibrosis. Am J Pathol 2016, 186:2232-2234

49. He J, Li X, Luo H, Li T, Zhao L, Qi Q, Liu Y, Yu Z: Galectin-3 mediates the pulmonary arterial hypertension-induced right ventricular remodeling through interacting with NADPH oxidase 4. J Am Soc Hypertens 2017, 11:275-289 